
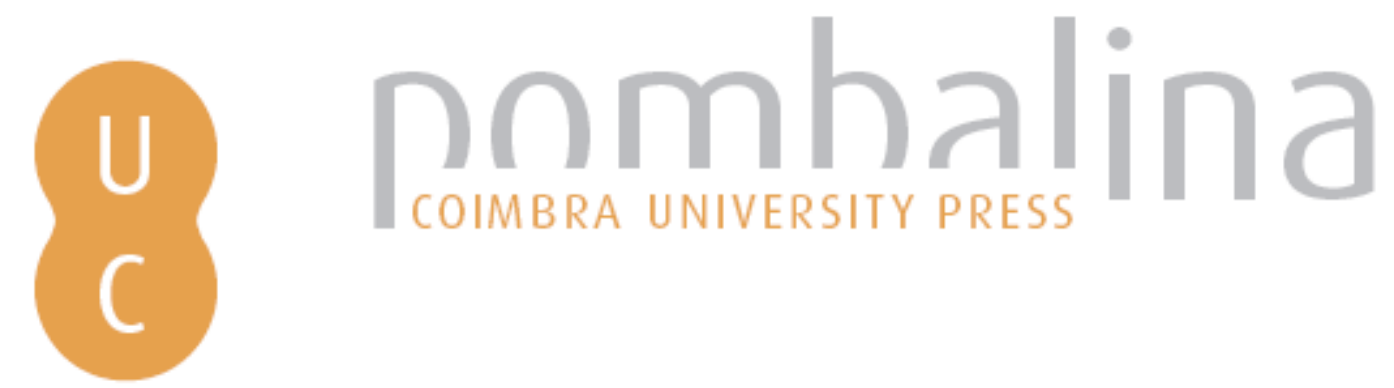

\title{
Producción y consumo de información científica en las Ataxias Raras con causa genética identificada (2003-2007)
}

Autor(es): $\quad$ Serrano-López, Antonio Eleazaar; Martín-Moreno, Carmen

Publicado por: Imprensa da Universidade de Coimbra

URL

persistente: URI:http://hdl.handle.net/10316.2/31962

DOI: $\quad$ DOI:http://dx.doi.org/10.14195/978-989-26-0319-3_38

Accessed : $\quad$ 26-Apr-2023 16:29:02

A navegação consulta e descarregamento dos títulos inseridos nas Bibliotecas Digitais UC Digitalis, UC Pombalina e UC Impactum, pressupõem a aceitação plena e sem reservas dos Termos e Condições de Uso destas Bibliotecas Digitais, disponíveis em https://digitalis.uc.pt/pt-pt/termos.

Conforme exposto nos referidos Termos e Condições de Uso, o descarregamento de títulos de acesso restrito requer uma licença válida de autorização devendo o utilizador aceder ao(s) documento(s) a partir de um endereço de IP da instituição detentora da supramencionada licença.

Ao utilizador é apenas permitido o descarregamento para uso pessoal, pelo que o emprego do(s) título(s) descarregado(s) para outro fim, designadamente comercial, carece de autorização do respetivo autor ou editor da obra.

Na medida em que todas as obras da UC Digitalis se encontram protegidas pelo Código do Direito de Autor e Direitos Conexos e demais legislação aplicável, toda a cópia, parcial ou total, deste documento, nos casos em que é legalmente admitida, deverá conter ou fazer-se acompanhar por este aviso.

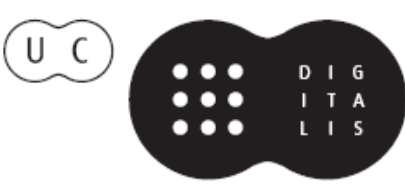


Maria Manuel Borges

Elias Sanz Casado

Coordenação

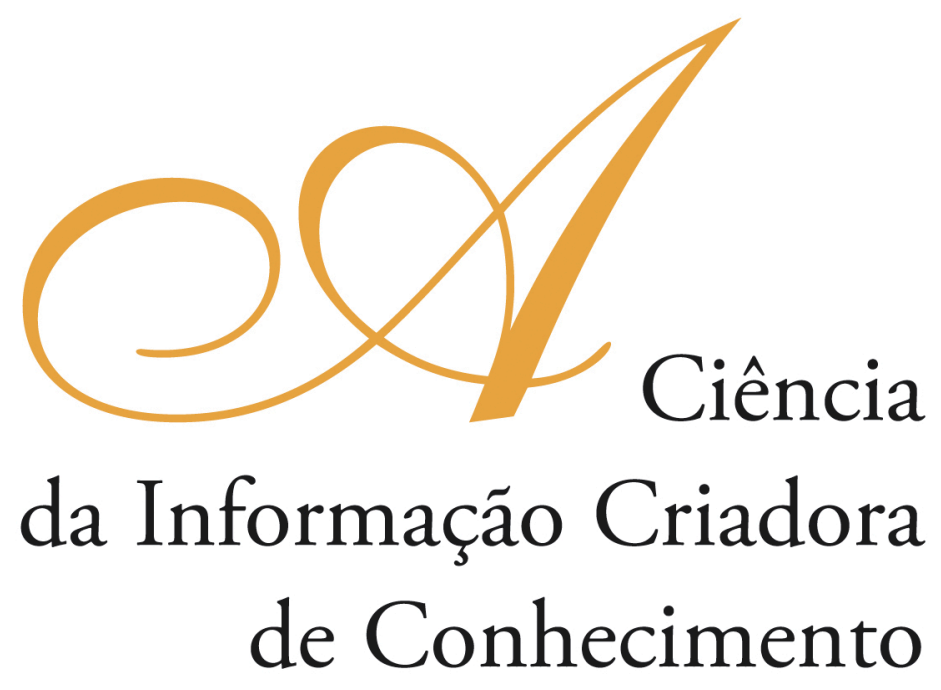

Vol. I

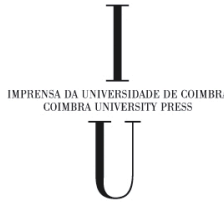

- COIMBRA 2009 


\title{
Producción y consumo de información científica en las Ataxias Raras CON CAUSA GENÉTICA IDENTIFICADA (2003-2007)
}

\author{
Antonio Eleazaar Serrano-López \\ Universidad Carlos III de Madrid (Espanha) \\ Carmen Martín-Moreno \\ Universidad Carlos III de Madrid (Espanha)
}

\section{Resumen}

El objeto de estudio de este trabajo es el de la producción y consumo de información científica en el área de las enfermedades raras o minoritarias y, dentro de ellas, muy concretamente, de las ataxias cerebelosas, que se puede definir como un trastorno caracterizado por la disminución de la capacidad de coordinar los movimientos. Para obtener los datos se han interrogado las bases de datos SCI y Medline y se han calculado los principales indicadores bibliométricos (recuentos de producción, obsolescencia, dispersión, tipología y capacidad idiomática) que nos han permitido determinar el perfil de la investigación en este tipo de patologías. Los resultados obtenidos nos muestran un creciente interés y un claro perfil multidisciplinar en la investigación de las ataxias cerebelosas.

\begin{abstract}
The main object of this research is the production and use of scientific information in area of rare diseases, and specifically, the cerebellar ataxias. We can define this disorder as a pathology characterized by a lack of coordination in movement. For data acquisition we use SCI and Medline databases and calculate the main bibliometric indicators (amount of production, obsolescence, dispersion, documental typology and idiomatic capacity) that allow us to establish the research profile on these pathologies. The results show us an increasing interest and a clear multidisciplinar y profile on cerebellar ataxias.
\end{abstract}

\section{Introducción}

En España, la realización de estudios métricos para conocer la actividad investigadora en diversos campos científicos, así como aspectos relativos a la investigación científica on de gran interés y se incrementan cada año los trabajos que se publican en revistas internacionales, sobre todo los pertenecientes a ciencias puras y experimentales atraen la atención de los investigadores de la bibliometría, que las analizan en profundidad y desde distintas ver tientes. En éste sentido, uno de los campos tradicionalmente más estudiado ha sido el de la medicina, en el cual contamos con multitud de trabajos relativos a la producción y consumo de información por par te del colectivo investigador, así como de las fuentes de información más utilizadas, que en este caso serían las publicaciones periódicas. 
En concreto el objeto de estudio de este trabajo es el análisis de la producción y consumo de información científica en el área de las enfermedades raras o minoritarias y, dentro de ellas y muy concretamente las ataxias, que podemos definir como un trastorno caracterizado por la disminución de la capacidad de coordinar los movimientos.

El concepto de Enfermedades Raras (ER) se acuña por primera vez a mediados de los años 80 en los Estados Unidos de Norte América (EEUU) y siempre estrechamente relacionado con el concepto de medicamentos huérfanos. Ambos términos se desarrollan en paralelo y van dirigidos a dar solución a los problemas que tienen las enfermedades de baja prevalencia.

La principal característica de las enfermedades raras consiste en su prevalencia, es decir, el número de casos estimado que tiene una enfermedad concreta. En la Unión Europea se considera una enfermedad rara cuando tiene una tasa prevalencia menor de 5 casos por cada 10000 habitantes. En segundo lugar, se trata de enfermedades graves o muy graves, crónicas, degenerativas, habitualmente mortales, y que frecuentemente conllevan una discapacidad. Ello supone una baja calidad de vida de los pacientes, que suele redundar en problemas psicológicos para ellos y su familia. Por último, la mayoría se manifiestan durante la infancia (aunque también pueden afectar a adultos), suelen ser enfermedades incurables y a menudo sin tratamiento eficaz, aunque en ocasiones existen tratamientos paliativos que mejoran la calidad y la esperanza de vida de estos pacientes.

En concreto las enfermedades en las que se centra este trabajo son las ataxias consideradas raras y que poseen una causa genética identificada. Debemos especificar que la ataxia como tal no se refiere a una enfermedad concreta, sino a un síntoma o conjunto de síntomas que se manifiestan como la falta de coordinación de las partes del cuerpo humano. Puede aparecer en las extremidades, el cuerpo, los ojos, etc. y habitualmente se asocia a las ataxias cerebelosas, un grupo de enfermedades neurodegenerativas que se caracterizan por una disfunción cerebelosa, sola o en combinación con otras anormalidades neurológicas (ROLÓN LACARRIERE et al., 2004).

Hoy en día la investigación en Enfermedades Raras se ha convertido en una de las principales prioridades en las políticas de salud de los países que componen la Unión Europea, y teniendo en cuenta la escasez de trabajos bibliométricos basados en el análisis de la producción y consumo de información que se han realizado sobre éstas enfermedades, consideramos de gran interés para la comunidad científica realizar estudios que permitan determinar las características de dicha investigación así como determinar los patrones que sigue, ya que de esta forma se puede tratar de predecir cual puede ser su posterior evolución.

El objetivo principal es conocer las características de la investigación, que ha dado lugar a publicaciones, en el campo de las ataxias clasificadas como raras con causa genética identificada.

Como objetivos específicos se estudian los hábitos de uso de información de los investigadores en éste área con el fin de determinar cuáles son los frentes de investigación y el perfil de consumo de información en la investigación acerca de estas enfermedades. 


\section{Metodología}

La cobertura temporal escogida para este estudio es el periodo 2003-2007, que nos ha permitido estudiar la tendencia que ha experimentado la investigación sobre estas patologías en los últimos años y observar su fluctuación en función de determinados factores.

La elección de las enfermedades que son objeto de este estudio, se llevó a cabo a través de la lista de enfermedades raras con causa genética identificada, facilitada por el INSTITUTO DE INVESTIGACIÓN DE ENFERMEDADES RARAS DE BASE GENÉTICA (2009). De este conjunto de enfermedades seleccionamos aquellas que nos interesaban, centrándonos en el caso concreto de las ataxias cerebelosas. La lista de patologías utilizadas para este trabajo es la siguiente:

- Friedreich ataxia

- Machado-Joseph Disease

- Spinocerebellar Ataxia 1

- Spinocerebellar Ataxia 2

- Spinocerebellar ataxia 6

- Spinocerebellar ataxia 7

- Spinocerebellar atrophy

Las bases de datos bibliográficas han supuesto las principales fuentes de información. Se han utilizado las bases de datos Medline y Science Citation Index (SCI) de Thomson Reuters. Medline debido a que está especializada en medicina y porque recoge la práctica totalidad de los trabajos publicados en este área, y SCI debido a la necesidad de contar con datos acerca del consumo de información científica. Se descartó la utilización de bases de datos nacionales, como el Índice Médico Español (IME), debido en primer lugar a que se trata de un estudio de marcado carácter internacional, y en segundo lugar porque las bases de datos nacionales no ofrecen información lo suficientemente actualizada y completa como para ser de utilidad en este estudio.

Para obtener los datos necesarios, se diseñaron para ambas bases de datos sendas estrategias de búsqueda que nos permitieran recuperar la información de la forma más exhaustiva posible, procurando que la información obtenida de ambas bases de datos fuera coherente. Para ello las estrategias de búsqueda debían ser lo más completas posibles y al mismo tiempo similares entre si, pues de otro modo estaríamos sesgando la información en cualquiera de las dos fuentes.

Se optó por no utilizar el tesauro MeSH que ofrece Medline porque no cuenta con términos específicos para cada una de las ataxias. Así, mientras la Ataxia de Friedich y la Enfermedad de Machado-Joseph si que cuentan con sus propios términos, el resto de ataxias cerebelosas se encuentran recogidas bajo el término "Spinocerebellar Ataxias", que incluye tanto las ataxias que son objeto de este estudio como otras patologías que no lo son. Por tanto, para no comprometer la precisión de los datos recuperados se optó por llevar a cabo las búsquedas sobre todos los campos recogidos en la base de datos.

Con los datos obtenidos a partir de dichas estrategias de búsqueda se construyeron dos bases de datos que han sido utilizadas para calcular los indicadores de producción y consumo que se presentan en los resultados. 


\section{Resultados y discusión}

\subsection{Indicadores de producción}

En primer lugar presentamos la distribución temporal de los trabajos objeto de nuestro estudio en las dos bases de datos utilizadas. Tras eliminar aquellas referencias en las que no contábamos con fecha de publicación o ésta se encontraba incompleta presentamos la siguiente distribución de frecuencias.

Tabla 1 - Trabajos por año de publicación y base de datos

\begin{tabular}{|c|c|c|}
\hline Ańos & No trabajos Medline & No trabajos SCI \\
\hline 2003 & 204 & 152 \\
\hline 2004 & 251 & 190 \\
\hline 2005 & 222 & 158 \\
\hline 2006 & 203 & 197 \\
\hline 2007 & 235 & 200 \\
\hline Total & 1115 & 897 \\
\hline
\end{tabular}

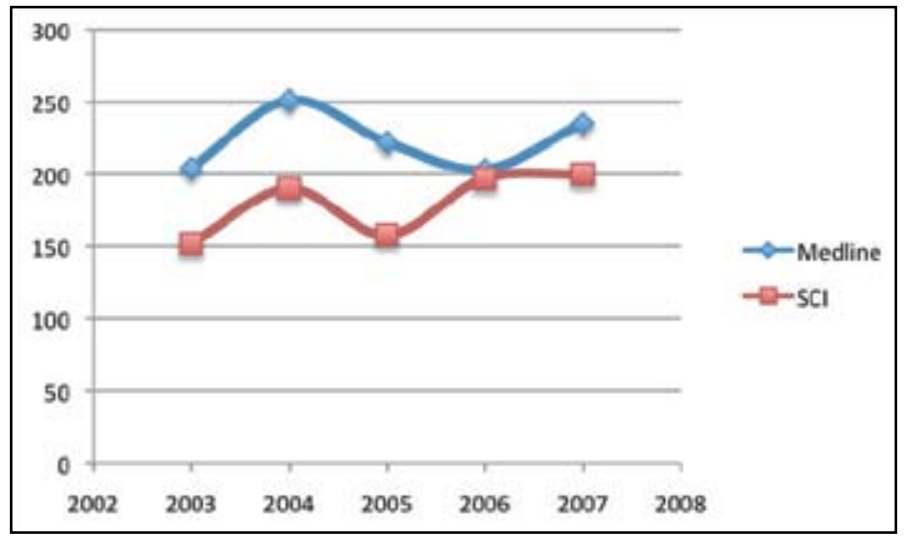

Fig. 1 - Trabajos por año de publicación y base de datos

Como se puede observar en la tabla 1 y la figura 1, los resultados en las dos bases de datos siguen una distribución muy parecida. La única diferencia a destacar es la que se produce en el año 2006, cuándo decrece el número de trabajos recogidos por Medline y sin embargo aumenta el del Science Citation Index (SCI). Este hecho puede deberse principalmente a la inclusión de un gran número de revistas por parte de Thomson-Reuters en sus bases de datos en el ańo 2007 (TESTA, 2009), por lo que necesariamente se vio aumentada la producción para el año 2006. Concretamente, gran parte de la inclusión de nuevas revistas se llevó a cabo en el área de ciencias médicas, algo que explica completamente el aumento en la producción de trabajos para el año 2006 en el SCI. 
Además, aunque en nuestro trabajo no hemos comparado la producción en estas enfermedades con la producción sobre trastornos neurológicos más comunes, si que existen estudios que han determinado la existencia de una mayor producción científica sobre enfermedades neurológicas raras que sobre aquellas más habituales, algo que los propios investigadores justifican por el hecho de que los trastornos más comunes cuentan con una etiología y prognosis bien definidas, si bien los autores del trabajo apuntan hacia otras razones (modas, mayor facilidad para obtener financiación, etc.) cómo las razones para este mayor interés por parte de los investigadores en neurología y neurociencia (AL - SHAHI et al., 2001).

En cuanto a la tipología documental en la que se han publicado los trabajos estudiados, mientras que en la base de datos Medline se trata en su totalidad de artículos de revista, en el Science Citation Index seguía la distribución que se recoge en la figura 2. En el gráfico podemos comprobar que la mayor parte de los trabajos son artículos de revista, mientras que el resto se distribuye entre actas de congreso y revisiones, con una pequeña parte repartida entre cartas, material editorial y un grupo que hemos denominado "otros" y que engloba correcciones, reprints, etc. Por tanto la distribución se ajusta al perfil clásico de los investigadores en ciencias de la vida y la salud (LOPEZ PIÑERO y TERRADA, 1992a).

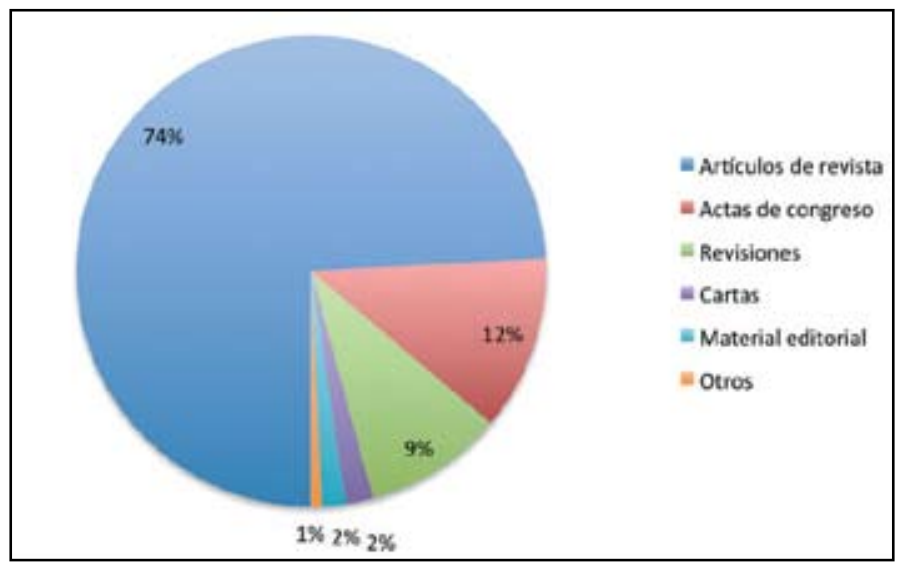

Fig. 2 - Tipologías documentales (SCI)

Por otro lado para presentar los resultados relativos a la capacidad idiomática de los científicos del área hemos elaborado la tabla 2. 
Tabla 2 - Distribución de los trabajos según su idioma

\begin{tabular}{|l|c|c|c|c|}
\hline & \multicolumn{2}{|c|}{ SCI } & \multicolumn{2}{c|}{ Medline } \\
\hline Idioma & Trabajos & Porcentaje & Trabajos & Porcentaje \\
\hline Inglés & 872 & $97,21 \%$ & 1057 & $92,40 \%$ \\
\hline Espańol & 11 & $1,23 \%$ & 13 & $1,14 \%$ \\
\hline Francés & 5 & $0,56 \%$ & 10 & $0,87 \%$ \\
\hline Alemán & 4 & $0,45 \%$ & 4 & $0,35 \%$ \\
\hline Chino & 2 & $0,22 \%$ & 16 & $1,40 \%$ \\
\hline Japonés & 1 & $0,11 \%$ & 27 & $2,36 \%$ \\
\hline Ruso & 1 & $0,11 \%$ & 4 & $0,35 \%$ \\
\hline Italiano & 1 & $0,11 \%$ & 1 & $0,09 \%$ \\
\hline Polaco & 0 & $0,00 \%$ & 3 & $0,26 \%$ \\
\hline Húngaro & 0 & $0,00 \%$ & 2 & $0,17 \%$ \\
\hline Noruego & 0 & $0,00 \%$ & 2 & $0,17 \%$ \\
\hline Portugués & 0 & $0,00 \%$ & 2 & $0,17 \%$ \\
\hline Checo & 0 & $0,00 \%$ & 1 & $0,09 \%$ \\
\hline Danés & 0 & $0,00 \%$ & 1 & $0,09 \%$ \\
\hline Rumano & 0 & $0,00 \%$ & 1 & $0,09 \%$ \\
\hline Total & 897 & $100,00 \%$ & 1144 & $100,00 \%$ \\
\hline
\end{tabular}

Cómo podemos observar en ambos casos el idioma mayoritario es el inglés, como era de esperar, sin embargo también podemos apreciar que la base de datos Medline ofrece una mayor cobertura para los trabajos publicados en idiomas asiáticos, como el Japonés o el Chino, y recoge también algunos otros idiomas que el SCI no contempla, cómo por ejemplo el Polaco, Portugués, Húngaro, etc. Además, cabe resaltar la buena posición en la que se sitúan los trabajos publicados en español, que podemos encontrar en el $4^{\circ}$ lugar en Medline y en el $2^{\circ}$ en el SCI, en ambos casos por delante del resto de idiomas europeos, salvo el inglés.

Para terminar con los indicadores de producción hemos utilizado medidas de dispersión para determinar qué revistas forman el núcleo de publicaciones más importantes en este área y cuyos resultados pueden observarse en las figuras 3 y 4.

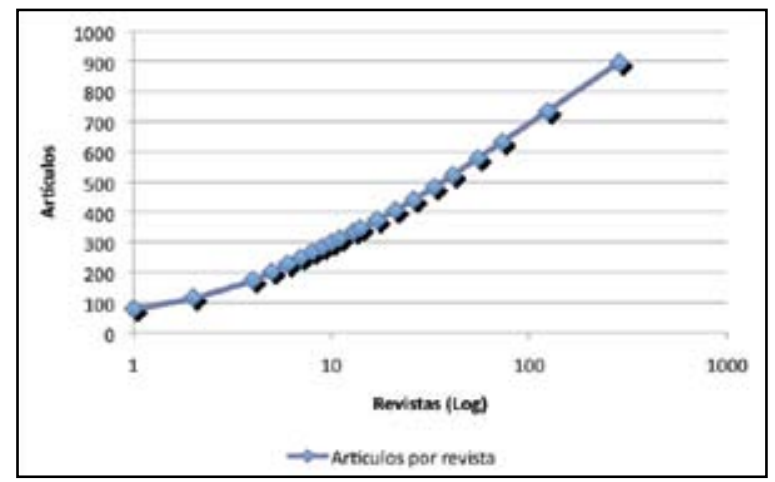

Fig. 3 - Gráfico semilogarítmico de dispersión (SCI) 


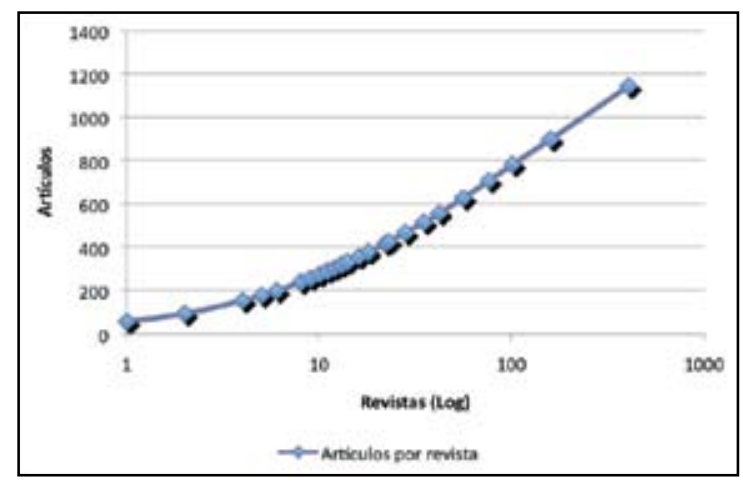

Fig. 4 - Gráfico semilogarítmico de dispersión (Medline)

En base a estos resultados podemos determinar que el núcleo de revistas para las bases de datos Medline y SCI está formado por 18 y 10 revistas respectivamente. Éstas se han delimitado mediante el método de las tres zonas, siendo considerado como núcleo el conjunto de revistas que acumulan aproximadamente el 33\% de los artículos.

Tabla 3 - Núcleo de revistas SCI y Medline

\begin{tabular}{|l|l|l|l|}
\hline \multicolumn{2}{|l|}{ SCI } & Medline \\
\hline Revistas & $\%$ art. publicados & Revistas & $\%$ art. publicados \\
\hline $\begin{array}{l}\text { MOVEMENT } \\
\text { DISORDERS }\end{array}$ & $8,92 \%$ & $\begin{array}{l}\text { Movement disorders : official } \\
\text { journal of the Movement } \\
\text { Disorder Society }\end{array}$ & $4,98 \%$ \\
\hline NEUROLOGY & $4,24 \%$ & Archives of neurology & $3,23 \%$ \\
\hline $\begin{array}{l}\text { JOURNAL OF } \\
\text { BIOLOGICAL } \\
\text { CHEMISTRY }\end{array}$ & $3,23 \%$ & Human molecular genetics & $2,62 \%$ \\
\hline $\begin{array}{l}\text { ARCHIVES OF } \\
\text { NEUROLOGY }\end{array}$ & $3,23 \%$ & Neurology & $2,62 \%$ \\
\hline $\begin{array}{l}\text { HUMAN } \\
\text { MOLECULAR } \\
\text { GENETICS }\end{array}$ & $3,12 \%$ & $\begin{array}{l}\text { Journal of the neurological } \\
\text { sciences }\end{array}$ & $2,01 \%$ \\
\hline $\begin{array}{l}\text { JOURNAL } \\
\text { OF THE } \\
\text { NEUROLOGICAL } \\
\text { SCIENCES }\end{array}$ & $2,90 \%$ & Journal of neurology & $1,92 \%$ \\
\hline $\begin{array}{l}\text { ANNALS OF } \\
\text { NEUROLOGY }\end{array}$ & $2,34 \%$ & $\begin{array}{l}\text { The Journal of biological } \\
\text { chemistry }\end{array}$ & $1,75 \%$ \\
\hline $\begin{array}{l}\text { JOURNAL OF } \\
\text { NEUROLOGY }\end{array}$ & $2,12 \%$ & Annals of neurology & $1,75 \%$ \\
\hline BRAIN & $1,78 \%$ & $\begin{array}{l}\text { Journal of neurology, } \\
\text { neurosurgery, and psychiatry }\end{array}$ & $1,57 \%$ \\
\hline $\begin{array}{l}\text { JOURNAL OF } \\
\text { NEUROLOGY } \\
\text { NEUROSURGERY } \\
\text { AND } \\
\text { PSYCHIATRY }\end{array}$ & $1,67 \%$ & Brain : a journal of neurology & $1,49 \%$ \\
\hline
\end{tabular}


La tabla 3 recoge las revistas que conforman el núcleo del SCI así como las diez primeras del núcleo de Medline. Los nombres de las revistas están recogidos tal y como aparecen en las bases de datos y, como se puede observar, aquellas situadas en los diez primeros puestos en Medline se corresponden con las que componen el núcleo del SCI, aunque en diferentes puestos. Por tanto, podemos determinar que se trata de las revistas más atractivas para los investigadores en el área de ataxias cerebelosas.

\subsection{Indicadores de consumo}

Mediante el análisis de las referencias incluidas en los trabajos recogidos en el Science Citation Index, hemos calculado una serie de indicadores que nos permitan conocer cómo es el consumo de información científica por parte de los investigadores en el área de las enfermedades de las que se ocupa el estudio.

En primer lugar, para calcular la obsolescencia de la literatura científica en este área se han calculado tanto la Vida Media (BURTON y KEBLER, 1960) como el Índice de PRICE (1973), que arrojan los siguientes resultados:

El Índice de Price: Tal como indican LOPEZ PIŃERO y TERRADA (1992b), consiste en el tanto por ciento de referencias de menos de cinco años, que llegaría al $50 \%$ en las "hard sciences" propiamente dichas, con componentes muy elevados de literatura efímera, y descendería a medida que aumenta la proporción de literatura clásica. En este caso el porcentaje de citas en los últimos 5 años es del $22.44 \%$ lo que nos hace pensar que se trata de un área a medio camino entre las disciplinas con literatura más efímera y las que utilizan literatura más clásica, lo cual queda confirmado cuando calculamos la vida media, que se da como resultado 7.89 años.

En cuanto a la dispersión de estas citas en las revistas científicas, al igual que en el caso de la producción hemos procedido a distribuir las citas y las revistas conforme al modelo de BRADFORD (1934), que se cumple claramente (figura 5) y nos permite distinguir las tres zonas habituales (núcleo, recta e inflexión de GROSS (1967)). El núcleo lo hemos determinado de nuevo mediante el método de las tres zonas, estableciendo que se encuentra compuesto por un total de 8 revistas que acumulan el $33.28 \%$ de las citas (un total de 11775 citas).

En este caso las revistas que componen el núcleo son las que muestra la tabla 4 y se corresponden principalmente con las áreas de genética y neurologíaneurociencia, con tan sólo una revista multidisciplinar (Proceedings of the National Academy of Sciences of the United States of America) entre las más citadas. Cabe destacar que, estableciendo una comparación con las revistas que forman el núcleo de producción (tabla 3, tan solo coinciden cuatro revistas. Incluso aquella que aparece como la más productiva tanto en el SCI como en Medline (Movement Disorders) no aparece entre las más citadas, lo que nos hace plantearnos que, a pesar de ser la revista más productiva, no recoge los trabajos con mayor impacto en este área. 


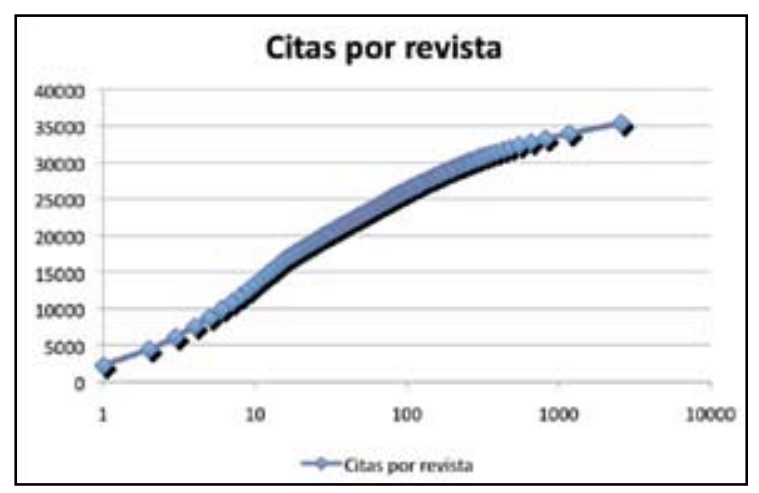

Fig. 5 - Gráfico semilogarítmico de dispersión de las citas bibliográficas (SCI)

Tabla 4 - Núcleo de revistas citadas

\begin{tabular}{|l|c|}
\hline Revista & \% Citas recibidas \\
\hline HUM MOL GENET & 6,28 \\
\hline NAT GENET & 6,24 \\
\hline NEUROLOGY & 4,80 \\
\hline J BIOL CHEM & 4,01 \\
\hline ANN NEUROL & 3,37 \\
\hline P NATL ACAD SCI USA & 3,36 \\
\hline AM J HUM GENET & 2,66 \\
\hline CELL & 2,57 \\
\hline
\end{tabular}

\section{Conclusiones}

Los investigadores que trabajan en el tipo de enfermedades estudiadas se sirven principalmente de las publicaciones periódicas para dar a conocer y consumir información científica y utilizan de forma mayoritaria el inglés para sus trabajos.

La distribución de los trabajos por publicación cumple la Ley de Bradford, tanto a nivel de producción como de consumo de información científica, por lo que un pequeño número de revistas acumula la mayor par te de la producción de trabajos científicos y las citas recibidas por éstos. Este grupo de publicaciones está formado casi por completo por revistas de las áreas de Neurología-neurociencia y genética. Por lo que podemos considerar que la investigación en el grupo de enfermedades estudiado posee un marcado carácter interdisciplinar.

Los indicadores de obsolescencia nos muestran unos resultados medios, probablemente debido a las diferencias en la vida media de las diferentes disciplinas involucradas en la investigación. 


\section{Referências bibliográficas}

Al-Shahi, R., Will, R. G., \& Warlow, C. P. (2001). Amount of research interest in rare and common neurological conditions: bibliometric study. British Medical Journal, 323.

Bradford, S. C. (1934). Sources of information on specific subjects. Engineering, 137.

Burton, R. E., \& Kebler, R. W. (1960). The half-life of some scientific and technical literatures. American Documentation, 11(1), 18-22.

Gross, O. V. (1967). Bradford's law the Keenan-Atherton data. American Documentations, 18, 46.

Lopez Piñero, J. M., \& Terrada, M. L. (1992a). Los indicadores bibliométricos y la evaluación de la actividad medico-científica. (III). Los indicadores de producción, circulación y dispersión, consumo de la información y repercusión. Medicina clínica, 98(4), 142-148.

Lopez Piñero, J. M., \& Terrada, M. L. (1992b). Los indicadores bibliométricos y la evaluación de la actividad medico-científica. (II). La comunicación científica en las distintas áreas de las ciencias medicas. Medicina clinica, 98(3), 101-106.

Price, DJS. (1973). Hacia una ciencia de la ciencia. (Lopez Piñero, JM, Ed.). Ariel.

Rolón Lacarriere, O, Rasmussen Almaraz, A, Hernández Cruz, H, Carranza del Río, J, González Cruz, M, \& Gutiérrez Moctezuma, J. (2004). Ataxia espinocerebelosa tipo 7: descripción de una familia mexicana. Revista De Neurologia, 38(8), 736-740.

Testa, J. (2009). Expansion Essay - Science - Thomson Reuters. Recuperado Julio 10, 2009, a partir de http://isiwebofknowledge.com/products_tools/multidisciplinary/webofscience/ contentexp/expansionessay/.

Instituto de Investigación de Enfermedades Raras de Base Genética. (2009). INERGEN. Recuperado Marzo 23, 2009, a partir de http://inergen.retics.net/inergen/home.aspx 